\title{
Theater: autonom und sozial
}

\section{Gerd Koch}

\begin{abstract}
Zusammenfassung
Im Folgenden werden sieben bildungs- und theater-philosophische Aufmerksamkeitsrichtungen skizziert. Sie sollen das Motto „Keine Angst vor ,Instrumentalisierung' und ,Verzweckung “' entfalten - und werden zeigen, wie vielfältig das Feld ist, das wir Theater nennen. Dabei bleibt hier der Begriff „Theater" unspezifisch (vgl. Schanze 2001). Gemeint sind performative, darstellende, recherchierende, zeigende, verkörpernde, handelnde Künste und Vorgänge, die mit der Verabredung des ,Als-ob', der Irritation und Widersprüchlichkeit, der Repräsentation sowie der Präsentation arbeiten und ein „Theatralitätsgefüge“ (nach Münz 1998) bilden, das zwischen Kunst-Theater und Alltags-Theater oszilliert. Theaterleute (im weiteren Sinne - also auch PädagogInnen, die sich und ihren Beruf ,theatralisieren') haben Methoden, um im Theater-Medium über sich und von sich zu handeln! Hier können Erkenntnisse gestaltet und ,in Szene gesetzt werden. Und es können Erkenntniswege, Interessen, Zwecke und die Einsprüche anderer dagegen - gezeigt werden.
\end{abstract}

Motto: Keine Angst vor ,Instrumentalisierung' und ,Verzweckung‘.

Nur Mut!

\section{Mut zur Verzweckung}

Im Rahmen der Sozialpädagogik - aus deren theaterpädagogischem Arbeitsfeld komme ich - stellt sich - wohl ganz ähnlich wie in der Fremdsprachendidaktik - die Frage, ob sich (das?) Theater für bestimmte Zwecke instrumentalisieren lässt oder aber als Kunst autonom sei und sich dem widersetzt bzw. widersetzen soll...

Das Wort von der Verzweckung ist kein philosophischer, analytischer, systematischer Begriff, sondern ein politischer Kampfbegriff, der seinerseits, befragt werden sollte, etwa so, wie es Bertolt Brecht (1992: 426, Kursivsetzung im Original) generell für Aussagen, für Sätze empfahl:

(1) Wem nützt der Satz?

(2) Wem zu nutzen gibt er vor?

(3) Zu was fordert er auf?

(4) Welche Praxis entspricht ihm?

(5) Was für Sätze hat er zur Folge? Welche Sätze stützen ihn?

(6) In welcher Lage wird er gesprochen? Von wem? 
Also: „Nur Mut!“ beim Denken und Handeln aus dem Theatralen heraus. Im Einzelnen führe ich etwas aus zum Doppelcharakter von Theater(kunst), heterogener Geselligkeit, Theater als kommunikativer Kultur, zum pädagogischen, forschenden Ansatz beim Szenischen, verwunderlich etwas auf den Punkt bringen sowie zum Forschen vom Handeln her gestalten.

Für meine Darstellung verwende ich ein Format, das sowohl wissenschaftspublizistisch wie ästhetisch/literarisch bedeutsam/nützlich ist: nämlich die Montage, den Collage-Essay, wie sie u.a. der Filmtheoretiker wie -praktiker Sergej M. Eisenstein und der Theaterreformer Erwin Piscator (zur ,politischintellektuellen Montage' und zur ,Montage der Attraktionen') sowie Richard Faber $(2005,1999)$ als wissenschaftlicher Darstellungsform und zur kritischmedialen Reportage sowie zum intellektuellen Erzählungs-Essay nach Walter Benjamin empfohlen haben.

\section{Doppelcharakter von Theater(kunst)}

Ich lasse mich generell leiten von Theodor W. Adornos Satz vom „Doppelcharakter der Kunst (hier: des Theaters, Anm. GK) als autonom und als fait social“ (Adorno 1970: 16), also als autonome wie soziale Tatsache(n). Ich lese diesen Satz Adornos dialektisch: Das Wort und zwischen Autonomie und Sozialem, zwischen sozial und autonom formuliert ein Wechselverhältnis; nichts, was sich ausschließt. Anders gesagt: Autonomie ist ein Aspekt des sozialen Vermögens von Theater. Und das Soziale ist eine autonome Kraft des Theaters. Beides kann produktiv zu einem empathischen, aber auch zu einem nicht minder schöpferischen strittigen Verhältnis führen.

Also, wir befinden uns bei Kunst und Theater im Feld von (An-)Spannung, (Ab-)Arbeit(ung) zwischen zwei Eigensinnen, bemerken Resilienz (WiderStandsfähigkeit), ein Begegnungsvermögen [was das Gegnerische in sich (aus-)trägt], eine Praxis, eine Poesie, eine Poetik - d. h. eine schaffende, hervorbringende Aktivität.

\section{Heterogene Geselligkeit}

Theater gilt als die ,sozialste', geselligste Form der Künste: Theater ist maßlos, saugt soziale Wirklichkeiten auf und stiftet neue Wirklichkeiten, an denen man nicht ohne weiteres vorübergehen kann. Theater ist in diesem Sinne „Weltergänzung“ (nach Chawtassi 1972) und hat Zeitgenossenschaft in Öffentlichkeit. Es konstituiert sich in und mit Öffentlichkeit; denn teatron ist Schauplatz.

Die Subjekte im Theater sind gemeinhin gesellig, relational, also in Beziehung stehend und nicht isoliert. Das betrifft die Spielenden/Zeigenden wie die zuschauend Aktiven. Man ist im Austausch mit anderen, zeigt sich, nimmt sich wahr in Vielfalt und erkennt, dass man kein monolithisches Unteilbares (also: kein In-Dividuum) ist, sondern ein Dividuum (ein Teilbares; auch Mit-Teilbares). 
Rollen-Spiel-Vermögen, Rollen- und thematisches Repertoire werden Ausdruck von (eben: sozialer) Identität als Beziehungs- und nicht isoliertes Ich-Sein. Der Mensch erscheine nach außen hin zwar als eine Einheit, sei aber im Innern kampfdurchtobt - so Bertolt Brecht ${ }^{1}$.

Das Auf-der-Bühne-Agieren ist ein Differenz-zeigen-Können, ist eine Übung des Vermeidens der sog. „Identitätsfalle“ (nach Sen 2007, vgl. Kleve et al. 2003), die eine vorschnelle und reduktionistische Vereindeutigung darstellt. „Ich bin ein Anderer" (Arthur Rimbaud) bzw. ich bin mehr als nur eine $/ \mathrm{r}$... ich gehöre unterschiedlichen Kollektiven/Kulturen/Gruppen an - und ändere dieses auch während meines Lebens (auch gegen Widerstände; denn eine Identität wird mir häufig genug fremdbestimmt gesetzt, etwa, um mich besser ,verwalten zu können - das geschieht mittels Etikettierung/labeling im internationalen Kontext, wie im nationalen und auch z. B. in der schulischen Lerngruppe)

Erst im „so und anders" (wie der Theatermacher Bertolt Brecht sagt), in diversity, zeigt sich Menschsein im emphatischen Sinne. Der aus der Karibik stammende Romancier und Kultur-Theoretiker Édouard Glissant (vgl. 2005) weist in dieselbe Richtung, wenn er vom empirisch gesättigten Modell der creolité, also des Kulturell-Gemischtseins als Normalfall spricht. Keine (vermeintlich eindeutige) identitäts-festigende Tiefen-Verwurzelung, wie in Deutschland sehr beliebt ist zu denken; und wenn schon ein Wurzel-Denken, dann eines in der Fläche: als Gewebe, Textur, Geflecht, als Netz.

Denn, so sagt es polemisch-zugespitzt der renommierte Philosophiehistoriker Kurt Flasch:

Wir Menschen haben, was man uns auch einreden möge, keine Wurzeln. Ich bin wirklich erstaunt, wie in den letzten Jahren, letzten Jahrzehnten, diese Rede, worin wir alles verwurzelt seien, Mode geworden ist. Es gibt kaum eine Woche im Fernsehen, ohne dass man hört, irgendjemand sei in der türkischen oder islamischen Tradition verwurzelt, oder wir seien in der antiken Welt oder im Christentum verwurzelt. Der Mensch hat keine Wurzeln. Hätte Gott uns eine Wurzel verschafft, dann wären wir Bäume. Wir haben aber Beine bekommen, um hinzugehen und wegzugehen. Und das ist die einfache Wahrheit ... Also so etwas geht in den Kopf der Menschen und wird nicht kritisiert ... und ... Lessing (mahnt): Lasst euch das nicht gefallen. Ihr habt Beine zum Weglaufen, zum Hingehen, wie ihr es für richtig findet. Ihr habt keine Wurzeln. (Flasch 2010) ${ }^{2}$

Dynamiken, relationale Unsicherheiten und Spannungen umgeben uns und wir

\footnotetext{
${ }^{1}$ Etwa 1941/42 notiert Brecht: „D as Individuum erscheint uns immer mehr als ein widerspruchsvoller Komplex in stetiger Entwicklung, ähnlich einer Masse. Es mag nach außen hin als Einheit auftreten und ist darum doch eine mehr oder minder kampfdurchtobte Vielheit, in der die verschiedensten Tendenzen die Oberhand gewinnen, so daß die jeweilige Handlung nur das Kompromiß darstellt.“ (Anm. GK: Brecht verwendet eine süddeutsche Variante für das Geschlecht eines Kompromisses) (Brecht 1993: 690)

${ }^{2}$ Kurt Flasch in seiner Dankesrede am 2. 5. 2010 bei der Verleihung des Lessing-Preises für Kritik in Wolfenbüttel. Weitere Stichworte in diesem Denk-Kontext sind: aktuell „Hybridität“ oder schon früher „Multiversum“ (im Anschluss an Ernst Bloch) (vgl. Han 2005; Koch 1986).
} 
tragen siemitund in uns: „Heterogenitätals gesellschaftlicher Normalfall“(soder Schauspieler und Erziehungswissenschaftler Stefan Gad) - Mehrsprachigkeit, Mehrdeutigkeit, transitorisch Sein, öffentlich für sich und mit anderen die Stimme zu erheben, Sprache(n) zu (er-)finden, zu haben und sich handelnd zu bewegen - was für die politische Philosophin Hannah Arendt Ausdruck von republikanischem Verhalten ist: das lässt sich auf der Theater-Bühne üben, zeigen, performieren, variieren - also in Szene(n) setzen - darin kann und darf man auf dem Theater/auf der Bühne (im Theater-Spiel, in der TheaterPädagogik) mit geringeren emotionalen, kognitiven und/oder sozialen ,Kosten' als auf der Welt-Bühne auch probeweise scheitern (vgl. Arendt 1993).

Und ein Wortspiel passt hier: man kann sich (wer weiß wie!) aufführen! Das ist ein Vorgang, der Autonomie und Soziales zusammenbringt ...

\section{Theater als kommunikative Kultur}

Eine Lehr-Lern-Kultur kann mittels Theatralisierung, mittels Sozialrauminszenierung gestiftet werden - nicht allein in Schulklassen oder Lerngruppen sondern durchaus auch in Lehrerzimmern, auf Schulhöfen oder in der Institution der Schule als ganzer - hier kann der Theater-Gedanke die Sozio-Architektur befruchten: der Theatermensch als Organisationsentwickler (vgl. Gad 2010). Wenn es richtig ist, wie der Sozialpsychologe Erving Goffman (1983) sagt, das unsere „Selbstdarstellung im Alltag“ (im Original „The Presentation of Self in Everyday Life") nach der Maxime geschieht: „Wir alle spielen Theater“, dann können wir unseren beruflichen Alltag auch nach und mit den Lehren aus dem Organisations- und Kommunikationsmodell des Theaters beschreiben und gestalten - und nutzen dann die Synergie des Autonomie- wie des Sozialpotentials von Theater(kunst).

Am 10.10.1942 notiert sich Brecht (1973: 526):
Es „wäre auszuarbeiten das thema angewandtes theater, dh, es müßten ei- nige grundbeispiele des einander-etwas-vormachens im täglichen leben beschrieben werden sowie einige elemente theatralischer aufführung im privaten und öffentlichen leben, wie leute anderen leuten zorn zeigen ... wichtig die auch im privaten leben geübte gruppierung in den verschie- denen situationen. ... rolle der gruppierung bei der alltäglichen drama- tisierung der sozialen beziehungen - wo und wie sitzt der vorgesetzte?"

Aktive Beteiligung und soziale Verantwortung sind Bedingungen vitaler Demokratie als kommunikative Lebensform. Der zeitgenössische politische Philosoph Charles Taylor behauptet, den klassischen Demokratietheoretiker Alexis de Tocqueville (1805 - 1859) zitierend, dass der moderne Mensch in der Gefahr stehe, „sich gänzlich in die Einsamkeit seines eigenen Herzens einzusperren“. Taylor sagt es dann so: „die dunkle (es gibt wahrlich andere! Anm. GK) Seite des Individualismus ist eine Konzentration auf das Selbst, die zu einer Verflachung und Verengung des Lebens führt, das dadurch bedeutungsärmer wird und das Interesse am Ergehen anderer oder der Gesellschaft vermindert" 
(Taylor 1995: 10). In schön expressionistischer Diktion formuliert der Philosoph Ernst Bloch (1970: 18) solchen Befund: "Da geht einer in sich. Das bessert ihn, wie er meint. Doch das merkt niemand, bleibt er darin zu lange. Er tritt dann oft nur auf sich selbst herum.Ünd wiederum mit Hannah Arendt gesprochen: eine öffentliche vita activa kann mittels theatraler Kommunikation gestaltet und geübt werden. Dafür benötigen, entwickeln und riskieren wir szenisch Sprache, Sprechweisen, Vergewisserungsweisen im weitesten und im variablen Sinne. Theater wird zu unserem Zeitgenossen.

In solche Denk- und Praxisweisen von angewandter Wissenschaft und/oder auch von life science (vgl. Asholt/Ette 2010) ${ }^{3}$ können auch die pädagogischen, praxeologischen Disziplinen von Methodik und Didaktik eingebaut werden. So spricht etwa die Theaterwissenschaftlerin und -pädagogin Dorothea Hilliger (2006), die an der Hochschule der Künste Braunschweig lehrt, von „theaterpädagogischer Inszenierung“ - es wäre - vielleicht noch etwas ungewohnt auch von ,fremdsprachenpädagogischer Inszenierung' zu sprechen - siehe die von Almut Küppers, Torben Schmidt und Maik Walter (2011) herausgegebene, verdienstvolle Publikation, die schon im Titel von „Inszenierungen im Fremdsprachenunterricht" spricht und dazu viele gelungene Beispiele liefert. Der Begriff des Inszenierens, auch des Performierens oder des Probens (vgl. Sack 2011) spricht vielleicht deutlicher als der des Theaters das Gemeinte aus.

\section{Zum pädagogischen, forschenden Ansatz beim Szenischen}

Institutionalisierte Wissenschaft, Forschung und Bildung wissen manchmal gar nicht, was sie an einem weiten Theaterverständnis und an einer entfalteten Theaterpädagogik haben könn(t)en, nämlich Anreicherung von ErkenntnisMethoden und erweiterte Darstellung von Ergebnissen/Erkenntnissen. Und nicht zu vergessen: Der explorative, entdeckende, propädeutische und motivierende Anteil von Theater-Spiel: Fokussierung von Problemen und/oder als interventionistische Forschung/als eingreifendes Verhalten (nach Bertolt Brecht: in Verhältnisse eingreifend).

Theater und Theaterpädagogik selbst können Forschungs- und Darstellungsweisen sein oder diese zumindest an- und bereichern sowie kritisieren (vgl. Bülow-Schramm et al. 2008 und Hentschel et al. 2008). Qualitative Sozialforschung etwa, als action research, Handlungsforschung, als kommunikative Wissenschaft kann sich der Bilder-, Dialog-, Körper- und Raumsprache sowie der differenziert gestalteten Zeit(en) des Theatralen bedienen (vgl. Koch 1997). $\mathrm{Zu}$ nennen wären theatre for development als Modell der Erwachsenen- und Gesundheitsbildung seitens der WHO in afrikanischen, ländlichen Regionen: Musterszenen, die medizinische Information und Verhalten in Szene setzten.

3 Siehe dazu: http://www.uni-potsdam.de/romanistik/ette/lebenswissen.html und auch Narbutovic, Katharina; Stemmler, Susanne (2011), Koch (2001). 
Oder: zeitgeschichtliches Dokumentartheater oder das Muster von meet the press des englischen Cockpit Theatre in dem SchülerInnen Personen aus der Geschichte/dem Geschichtsunterricht zu einer inszenierten Pressekonferenz bitten. Auch das neue Format des „Theaterfeature“ wäre zu nennen:

Das Theaterfeature ist eine Synthese aus den wesentlichen Elementen des Radiofeatures und visuellen Methoden und Arbeitsweisen des Theaters. Seriös recherchierter Stoff zu einer bestimmten Thematik verbindet sich auf der Bühne mit dem handlungsorientierten Mitteln des Theaters [...]. Historische Elemente wie Dokumente, Lieder, Protokolle, Zeugnisse und Zeitungsartikel werden performativ in Szene gesetzt. Durch sprechchorische, solistische und körperbildliche Ergänzungen wird die geschichtlich oft sehr umfassende Thematik an die Zuschauer_innen herangetragen [...]. Die [...] Stückfassung ist das Ergebnis einer lang andauernden Recherche, von Probengesprächen und Erzählungen der Darsteller_innen und der sorgfältigen Montage von dargestellten Szenen, Musik-, Bild-, Originalton- und Videoeinspielungen. (Lassak 2011: 6)

Wir kennen im Theater wie in der Wissenschaft und in der Kriminalistik den Terminus recherche - also Suche; ja: Forschung. Hier zeigt sich eine Chance des Fächergrenzen überschreitenden Arbeitens!

Sinnliche, sinnlich-wahrnehmende, aus wechselnden Blickwinkeln (also: prismatisch. kaleidoskopisch) betriebene, auch dialogische Wissenschaft/Forschung, why not! Theodor W. Adornos Untersuchung mit dem Titel „Ästhetische Theorie“ wäre dann doppelsinnig zu lesen: als Theorie der Ästhetik und als eine Theorie, die sich der Ästhetik (aisthesis, Wahrnehmung, Sinnlichkeit) nicht entzieht.

Theater und Theaterpädagogik wissen manchmal noch gar nicht, dass und wie sie selbst Wissenschaft, Erkenntnis, Forschung und Bildung betreiben [siehe aber die temporären Akademien an einigen Theatern - z. T. auch und zum Glück manchmal recht schräge Unternehmungen (schräg zu sein war im übrigen eine Forderung an die Didaktik und Methodik der Schulfächer, die der Erziehungswissenschaftler Horst Rumpf in einer Podiumsdiskussion der Ständigen Konferenz Spiel und Theater an Hochschulen vor Jahren an der Pädagogischen Hochschule Ludwigsburg stellte - warum soll nicht ein Unterricht, im Schülerjargon gesprochen, ächt schräg sein?!)]. Der Physiker Niels Bohr meinte, wenn er nicht einigermaßen verrückt [ver-rückt, normabweichend, ,ex-zentrisch' (Helmuth Plessner)] gewesen wäre, hätte er wohl keine - neuen - Erkenntnisse erwirkt.

Theatrale, szenische, sinnliche Ansätze beugen Betriebsblindheit und Spezialisierung als Erfahrungseinschränkung vor. Schon 1979 formulierte es Horst Rumpf (vormaliger Gymnasiallehrer und später Erziehungswissenschaftler) so:

Der Ansatz beim Szenischen, bei der Arbeit von Subjekten an der Konstitution ihrer (auch symbolischen, GK) Erfahrungswelt kostet mehr Zeit als die Beschränkung auf den (sog., GK) puren Inhalt. Das gilt sowohl für die Forschungspraxis als auch und vor allem für die ihr vorgelagerte 
Erziehungs- und Lernpraxis. Probieren, Umwege, Irrwege bei der Erforschung der neuralgischen Punkte und Phasen von Lernprozessen sind (aber, Anm. GK) nicht als Zeitverlust zu verbuchen. (Rumpf 1979: 165)

Wir können von einer „Theatralisierung von Lehr-Lernprozessen“ (nach Koch 1995) sprechen. Lehren und Lernen sollte immer mehr zu einem variablen, konstruktiven Kommunikationsangebot werden. „Im lehren muß das lernen enthalten bleiben" - fordert Bertolt Brecht 1930 (zitiert nach Koch 2005: 197). Eine einlinige Kommunikation nach dem Sender-Empfänger-Modell sollte nicht länger bedient werden. Die Bühne, das Theater als Orte szenischen und auch informellen Lernens und Lehrens können als Modell für schulisches Lehren und Lernen genutzt werden. Horst Rumpf (2010) wendet sich „[g]egen die Verkürzungen des etablierten Lernbegriffs“. Der Philosoph Peter Bieri (2011) - als Roman-Autor: Pascal Mercier (z.B. Nachtzug nach Lissabon) - nutzt Theater-Anleihen zur Beantwortung der Frage „Wie wollen wir leben?“

Ein pädagogisches Denken in szenischen Theatraliäts-Kategorien führt auch dazu zu bemerken, dass Wissen nicht nur über die sog. Inhalte tradiert wird, sondern ganz häufig (a) durch die Formen, in denen es erscheint (Rancière 1994), (b) durch die Haltung des Forschenden, der (s)ein Wissen ausbreitet (oder dem man es entreißen muss, wie Bertolt Brecht erzählt) sowie (c) durch die mediale Bearbeitung von Wissen. Und (d): Szenisches Gestalten (re-)aktiviert häufig erst Wissen bzw. ent-deckt schlummerndes, verdecktes Wissen: Daten, Fakten, Strukturen, Gefühle, Strategien, Erfolge/Misserfolge kommen als Potenziale ans Tageslicht (es entsteht eine Reihung von Potenz Kompetenz - Performanz).

Exemplarisches Lernen und Lehren kann verbunden werden mit theatraler/szenischer Phantasie (vgl. Negt 1971) und es ließe sich spielen mit der Abkürzung „TZI“ - was das Kürzel für eine alltagsweltliche, kommunikative Therapie und Selbsthilfe ist, die von Ruth Cohn entwickelt wurde: „Themenzentrierte Interaktion". In ihr sollen das Thema, das Ich und das Wir ein dynamisches, aktives Dreiecks-Verhältnis bilden - umrahmt vom globe, d. h. Thema, ich und wir sind infiltriert von unserer Umgebung, wir sind ,porös ${ }^{c}$ (vgl. Buck-Morss 2011: 150ff.), so dass in uns immer auch anderes eingelassen wird - also wir sind Teil eines globe theatre/théâtre universel - auch dort, wo ,ich' in kleinen, geschützten Interaktionsgruppen vermeintlich nur mit ,uns ${ }^{6}$ thematisch versammelt bin. Die drei Buchstaben TZI könnten auch stehen für: Theater-zentrierte Interaktion ... im global context.

Sind es nicht häufig Personen und ihre Performanz, die uns etwas sagen was ja doppelt lesbar ist: Sie sind diejenigen, die etwas sagen im Sinne von Zeigen und Erzählen, und sie sind die, die uns etwas bedeuten - nicht zuletzt durch ihre eigensinnige, auch farbig, unmittelbar erlebte Person, als Charakter, als Verkörperung und durch die Art und Weise ihres Kommunizierens. Faktizität und Geltung kommen in einem Vorgang, nämlich im Spiel- und InszenierungsVorgang, zusammen. Es entsteht ein pragmatisches, alltagsweltlich grundiertes Mischungsverhältnis zweier Mentalitäten - eine verweist in Richtung Autonomie, Nonkonformität und Eigensinn, die andere in Richtung sozial und 
kommunikativ. Die angelsächsische, pragmatistische Tradition von Pädagogik formuliert das methodische Verhältnis Drama, Szene, Theater in etwa solchem Sinne:

We call it Drama in Education [...] There are a variety of names [...]: Theatre in Education, Theatre Education (without the 'in'), Drama Education, Drama Work, Creative Drama. It depends whether it's informal, whether it's an educational context. Sometimes you just call it theatre." (Shirley Harbin). Stig A. Erikson erklärt, in Norwegen würde die Sphäre der Aktivitäten dessen, was man hierzulande mit Theaterpädagogik bezeichnet, “drama pedagogy' or ,drama in education'; and in everyday language just drama" heißen. "We don't say: You must do a play. What we say is: understand or participate in theatre arts." (Shirley Harbin). "Unser Ziel war es, unseren Studenten, Kindern, Erwachsenen (mit "creative drama", "creative play", Anm. GK) die Möglichkeit zu geben, sich selbst mit Hilfe von dramatischen respektive darstellenden Formen auszudrücken. (Nora Roozemond, zit. nach Koch 2008).

Auch solche Sichtweise ist möglich: Drama kann als ein Intensitätsgrad, der über alltägliche Normalitäts-Kommunikation hinausweist, verstanden werden, gleich einer herausragenden Bedeutung oder erheblichen Bedeutung = eine empörende Hebelwirkung habend, wie Vilém Flusser sagen würde:

Der Hebel ist nämlich eine sogenannte einfache Maschine, wer sich damit befaßt, hat es mit Mechanik zu tun, und das ist bekanntlich nicht nobel.[...] [Er jedoch will sich dem Thema widmen,] um zu sehen, was herauskommt, wenn man den Hebel als eine Maschine zum Empören aus der Niedertracht [man nehme diese Wortbilder ganz deutlich: Empören heißt, etwas Hochheben; Niedertracht bedeutet, daß etwas heruntergezogen wird, was durch Empörung, mittels Technik, Mechanik, Hebelwirkung wieder in die Höhe gebracht werden kann, Anm. GK], also als eine Maschine zur Menschwerdung ansieht. [...] Der Hebel ist eine Maschine. Das Wort Maschine (ebenso wie das Wort Mechanik) entstammt dem griechischen Mechanike, das etwa listige Vorrichtung bedeutet. Das Wort Machination zeigt, was gemeint ist [auch unser deutsches Verb ,machen', also etwas tun, etwas verändern, gehört zu diesem Wortstamm, Anm. GK.]: Nämlich z.B. das Trojanische Pferd, also eine Falle, in welche die Trojaner hineinfallen sollten. Ulysses, der Erfinder des Pferdes, heißt polymechanikos, was mit der Listenreiche übersetzt wird [eigentlich „Polymechanos“/По $\lambda \varepsilon \mu^{\prime} \eta \chi \alpha v o \zeta$, Anm. GK]. Demzufolge ist die Mechanik eine Strategie zum Überlisten. Wenn man den Hebel ansieht, dann kann man sehen, was die Mechanik überlistet. Nämlich das Schwere im Körper. Das erinnert an orientalische Kampfstrategien wie etwa Judo: Dort wird versucht, die Kraft des Feindes gegen diesen einzusetzen. Die Mechanik wendet die Gesetze der physikalischen Natur listigerweise so um, damit sie die Natur überwinden möge. Wer ein mechanisches Weltbild hat, der blinzelt listig. (Flusser 1991: 71) 


\section{Verwunderlich etwas auf den Punkt bringen}

Am Anfang zitierte ich Theodor W. Adornos Wort vom „Doppelcharakter der Kunst (hier: des Theaters, Anm. GK) als autonom und als fait social" als (m)eine dialektische Maxime. Im Laufe meiner Assoziationen habe ich den Strang der Autonomie der Kunst, des Theaters - vermeintlich - etwas aus den Augen verloren. Das will ich hier pointiert auszugleichen versuchen. Und mit dem Wort ,pointiert' habe ich schon ein Kriterium benannt, das m. E. ins semantische Feld von Autonomie gehört: Künstlerische Produktionen bringen etwas prononciert, also mit Betonung (siehe auch „Drama“ als Intensitätsmaßstab) auf den Punkt bzw. sie setzen einen - auch einen unerwarteten, ungewohnten. Oder einen, der den ihn setzenden Menschen mit seinem Interesse oder die Gruppe von pointierten Menschen nicht verschweigt (es findet dadurch - auch - eine Anthropomorphisierung statt) (vgl. Lukács 1972).

Künstlerische, theatrale Produktionen respektieren den Eigensinn der Beteiligten - das sind nicht nur Personen, sondern auch die einzelnen Medien, Gewerke, Arbeitsweisen, Materialien, Texte, Techniken, Stilelemente, die das Widersprüchlich-Ganze von Theater/Inszenierung/Aufführung ausmachen. Sie alle bilden - unter Wahrung ihrer Eigenständigkeit, also Autonomie/Eigengesetzlichkeit - den kreativen Raum - und sie vermögen, sich kreativ im Raum zu stoßen. Sie stellen häufig etwas Ungewohntes, Verwunderliches neu her und stellen es aus und zur Disposition. Das stiftet ihren Bildungswert. In Anlehnung an die Philosophin Hannah Arendt schreibt Marie Luise Knott:

Dadurch, dass die Sätze vom Handelnden her gedacht werden und keine ,objektiven' Beschreibungen und Urteile sind, können auch exotische, untergegangene (nicht siegreiche) Stimmen und Aspekte der Geschichte auf die Bühne geholt werden. Die besiegten Geschichten werden dem Vergessensein entrissen. Der Einzelne, der droht, überflüssig zu werden, erhält die Vorstellung zurück, dass es auf ihn [... ] ankommt, dass es sein Tun oder sein Vorstellen ist, das ,alles wenden“ kann. (Knott 2011: 112)

Künstlerische Arbeiten sind Zeitgenossen und müssen sich immer aufs Neue bewähren, sie können sich - nicht mehr - auf traditionelle Gepflogenheiten/Stile/Geschmacksrichtungen berufen. Der Kunst-Soziologe Hermann Pfütze (1999) sieht darin sogar idealtypisch eine Struktur-Ähnlichkeit mit Prinzipien demokratischer Politikgestaltung: immer aufs Neue muss Politik sich bewähren und kann sich nicht auf stillschweigendes Vererben etwa von Macht oder Konventionen ausruhen. Auch Kunst muss um ihre Relevanz/Anerkennung ringen, muss das Risiko des Nicht-verstanden-Werdens eingehen. Und steht es nicht ganz so mit wissenschaftlichen Erkenntnissen und Forschungen, Bildungsmaßnahmen? Kunst ist wie manche wissenschaftliche Erkenntnis in ihrer Autonomie-Dimension eine Störung des Ablaufs, eine Norm-Abweichung, ein Krisen-Signal, eine non-konformistische, ex-zentrische, eigensinnige $\mathrm{Zu}$ spitzung, eine Ent-Funktionalisierung, eine (Er-)Öffnung. Darin liegt auch ihre Sozialität. 
Zusammengefasst: Autonomie und Sozialität müssen selbstgesetzte, selbstgestaltete und selbstreflektierte Zwecke des Theatralen sein - keine aufgeherrschten, aufgedrängten. Das kommunikative Muster „Theater" ist in der Lage, couragiert eine Entzweckung fremdgesetzter Zwecke vorzunehmen!

Autonom-Sein heißt Selbst-Gesetzlich sein. Subjektiv zielt das etwa auf das Verhalten von mutigen, unabhängigen, hier: forschenden, erkennenden, lernenden, gestaltenden, auch spielenden Menschen. Objektiv zielt es auf die Wahrung/Würdigung des Eigenrechts von Gegenständen, Fragestellungen, die zur Klärung anstehen.

Klären und Gestalten kann man Zwecke von Kunst, von Theater, von Inszenierung nennen - aber sie sind eigen- und nicht fremd-gesetzte Zwecke gestaltet in Autonomie und in reflexiven, also bedachten sozialen Gefügen. Schaut man in Richtung auf (stark) institutionalisierte Lehr-Lern-Prozesse, wie sie paradigmatisch und traditionell der schulische Unterricht zeitigt, dann ist es hilfreich und realistisch, zwei gleichzeitig wirkende Netzwerke, in denen Schule/Unterricht/LehrerInnen- und SchülerInnen-Handeln stattfindet, wahrzunehmen in ihrer wechselseitigen (besser: dialektisch) Aufeinanderbezogenheit. So ist das Handeln eines Lehrers/einer Lehrerin innerhalb eines Schulsystems immer edukativ (also im engeren, unmittelbaren Sinne pädagogisch-kommunikativ) bestimmt und zugleich ist es kustodial (also organisierend, ordnend, sichernd; custos = Wächter) bestimmt (vgl. Rumpf 1971). Das führt zu Friktionen. Beide Netzwerke können nun flexibilisiert, modernisiert werden - indem sie theatralisert werden. Das Schlagwort von der ,Entschulung der Schule' steuert das gemeinte in der Tendenz richtig an (vgl. Koch et al. 1986).

\section{Forschen vom Handeln her gestalten}

Wenn Theater autonom und sozial ist, dann hat das Folgen für die Forschung, zum Beispiel für Wirkungsforschung, weil Eigensinn und sozialer Sinn gleichermaßen berücksichtigt werden müssen. Für das Soziale stellen die Sozialwissenschaften beschreibende, quantitative und qualitative Verfahren bereit. Insgesamt: Qualitative, alltagsweltliche/lebensweltliche Orientierungen Formen der Forschung sind vorzuziehen - aber sie sollten mit quantitativen durchaus verbunden werden (vgl. Flick et al. 2000).

Aber wie schaut es mit dem speziellen Aspekt der künstlerischen, kreativen, sinnlichen, ästhetischen, mentalen Autonomie aus? Wie dort (was?) erforschen? Alexander Mitscherlich (Psychoanalytiker und Sozialtheoretiker) prägte für dieses Aufgabenfeld den Begriff der Forschung durch Vergewisserung - also: man möge Wirksamkeitsdialoge (vgl. Appel et al. 2000) führen. Das sind Forschungen auf mittlerer Abstraktionsebene, die Interessen, Bedürfnisse Zweifel - also so genannte subjektive, weiche und auch werthafte Dimensionen noch durchscheinen lassen. Auf einer Skala von Universalität und Kontextualität würde sich solcher Forschungsansatz in der Nähe des Nicht-Verleugnens seiner Kontextualität finden und nicht in der Nähe einer möglicherweise durch 
abstrakte Zahlen gestifteten - vermeintlichen - Universalität.

\section{Ein Exempel aus der Forschung}

Eine empirische, erziehungswissenschaftliche und theaterpädagogische Untersuchung, unternommen mit der Methode der "grounded theory“ (also theoriegeleiteter Selbstthematisierung) mit dem Titel „Paedagogus Ludens“ (also: spielender Pädagoge) von Antonis Lenakakis (2003a) zeigt, dass eine allgemeine theatrale Verhaltensausstattung einer Lehr-Person ein job enrichment und job enlargement für viele pädagogische und methodische Eventualitäten bereithält - eine berufliche vita activa (Hannah Arendts Begriff hier aufgreifend). Lenakakis stellt seine Untersuchung „Zur Wirkung der Spiel- und Theaterpädagogik auf Lehrer(innen) “ im Überblick so dar, beginnend mit zwei Selbstaussagen der ,Beforschten`:

Ich habe als Lehrerin Sicherheit und Souveränität gewonnen, mit Situationen umzugehen" (sagte eine Sekundarstufen-Lehrerin). "Nicht immer nur auf ein bestimmtes geplantes Ergebnis zu schielen, sondern sich einfach zu trauen, auch neue Sachen auszuprobieren und die Schüler und allgemein den Menschen als ,Überraschungsei' anzusehen" (sagte eine weitere Sekundarstufen-Lehrer) und der Forscher Lenakakis fragt: „Wie erleben eigentlich Lehrerinnen-Spielleiterinnen und Lehrer-Spielleiter sich und ihre Arbeit?“ Seine Antwort im Überblick: „Es ist schon verwunderlich, dass es zu dieser Kernfrage der Spiel- und Theaterpädagogik keine Antwort geschweige denn empirische Untersuchungen gibt. Auf der anderen Seite gibt es viele spiel- und theaterpädagogische Aus-, Weiterund Fortbildungsangebote von staatlichen und nicht-staatlichen Einrichtungen sowie Universitäten in Deutschland, und jedes Jahr werden zahlreiche in diesem Feld ausgebildete Lehrkräfte in der Schule tätig. ( ) Im Rahmen einer qualitativ-empirischen Untersuchung habe ich die Selbstinterpretationen von spiel- und theaterpädagogisch ausgebildeten und tätigen Lehrerinnen und Lehrern dokumentiert, analysiert und daraus theoretische Aspekte entwickelt. Dabei interessierten mich vor allem die Handlungsmotive und Verhaltensweisen der Lehrerinnen und Lehrer bei der Realisierung eines spiel- und theaterpädagogischen Konzeptes, die fördernden Faktoren ihrer Arbeit, die Schwierigkeiten, die sie zu bewältigen haben, und die Strategien, mit denen sie arbeiten. ( - ) Am Beispiel von 17 befragten spiel- und theaterpädagogisch ausgebildeten und tätigen Berliner Lehrerinnen und Lehrern der Grund- und Sekundarstufe, die in der Untersuchung als Expert(inn)en ihres eigenen beruflichen Handelns betrachtet werden, werden Prozesse professioneller Entwicklung und Faktoren, die den Berufsalltag bestimmen, entdeckt und nachgezeichnet; im weitesten Sinne die Wirkung der Spiel- und Theaterpädagogik auf Lehrerinnen und Lehrer aus ihrer subjektiven Sicht empirischsystematisch, aber differenziert aufgezeigt. ( - ) Bei der Entwicklung und Formulierung der Fragestellung habe ich meinen Auftrag nicht nur darin gesehen, eine rein methodische Anreihung von Aufgaben oder eine präzise Formulierung der Ziele systematisch zu verfolgen, sondern auch die 
gegenstandsbezogenen Aspekte einer permanenten Revision zu unterziehen und darauf aufbauend empirisch begründete Modifikationen als quasi dynamischen und sich aus sich selbst heraus entwickelnden Entscheidungsprozess vorzunehmen. ( - ) Meine Haltung zum Untersuchungsfeld musste also hauptsächlich abduktiv sein, d. h. mein theoretisches Wissen habe ich bewusst nicht zu sehr in den Vordergrund treten lassen, damit ich gegenüber neuen Erkenntnissen offen sein konnte. (Lenakakis 2003b: 25f)

Soweit der Forscher Lenakakis (jetzt als Professor an der Aristoteles-Universität in Thessaloniki tätig). Lenakakis geht mit einem erziehungswissenschaftlichen und Lehrer- bzw. Schulforschungsblick an sein Thema, den „Paedagogus ludens", heran. Er prüft empirisch auf dieser Folie, inwieweit spiel- und theaterpädagogisches Vermögen von LehrerInnen hilfreich ist, die ,Welt der Schule (als Lebenswelt und Struktur) zu gestalten, zu bewältigen. In organisationssoziologischer Sprache gesagt: Inwieweit schafft spiel- und theaterpädagogische (Zusatz-)Qualifikation von LehrerInnen eine innovative schulische Betriebskultur? Der Autor bejaht die Kraft, wobei er - wie mir scheint und wie der Titel mit Akzent auf „ludens“ nahelegt - das TheaterSpiel und/oder TheaterSpiel als Potenz in diesen Prozessen würdigt.

Nicht die TheaterlehrerInnen (als spezialisierte LehrerInnen im Fach Darstellendes Spiel/Schultheater etwa) sind es primär, die sein Interesse hervorgerufen haben, sondern alle jene, die im Schulalltag, der durch den Verfasser beforscht wurde, nach spiel- und theaterpädagogischen Maximen didaktisch/methodisch generell Unterricht gestalten wollen - gewissermaßen ein Unterrichts- und Schulprinzip darin sehen.

Wählt man die Sprache der Modularisierung von Lehr-Lernprozessen, dann lässt sich sagen, Lenakakis spricht mit seiner erziehungswissenschaftlichen Untersuchung die Dialektik (oder das Aufeinander-Angewiesen-Sein) von Fachkompetenzen (subject-related competences) und sog. fachunabhängigen Kompetenzen (generic competences) an. Er sieht in der durch ein Studium der Spiel- und Theaterpädagogik erworbenen Kompetenz eine zusätzlich miterworbene Fähigkeit, Transferleistungen zu erbringen, also das gewonnene Wissen auch andernorts in Schule und Unterricht zu verwenden - auch in Hinblick auf eine Verbesserung von Arbeitszufriedenheit etwa.

Forschungsarrangements der Ethnologie, der empirischen Kulturwissenschaft mit ihren differenzierten, variablen (dichten) Beschreibungen (vgl. Geertz 1987) können ferner Pate stehen in solchen Untersuchungsfeldern, die der Forscher Lenakakis mittels „grounded theory“ erforschte. ${ }^{4}$ Auch ein gutes Feature (s. o. zum „Featuretheater) wäre eine wissenschaftlich-publizistische Untersuchungs-

\footnotetext{
${ }^{4}$ Siehe zur Wirkungsforschung „Zeitschrift für Theaterpädagogik. KORRESPONDENZEN“, 2006, Heft 48: 35-72. Im besonderen Pigl et al. (2006: 65-69) zu dem hier angesprochenen Zusammenhang einer Wirkungsforschung auf mittlerer Abstraktionsebene. In Bezug auf qualitative Sozialforschung und als Mittel zur Teilnehmer/innen orientierten Selbstverständigung siehe Koch (2012) zum „Erzähl-Café als Methode in der rekonstruktiven Sozialen Arbeit“.
} 
und Dokumentationsweise, die als forschende Vergewisserung zählen kann (vgl. Projektteam Lokaljournalismus 1986).

Es gibt ferner diese etablierten akademischen Forschungsmethoden, die zugleich einer Evaluation in anschaulicher Weise dienen können: action research/Handlungsforschung, hermeneutische Verfahren, Inhaltsanalyse, case studies/Fallstudien verbunden mit ratings, offene, problemorientierte Interviews, grounded theory-Forschung (s. o.), narrative lebensgeschichtliche Interviews, Biografieforschung, Dokumentenanalyse, Verlaufs- und LebensLern-Begleitungsforschung, Pfadforschung als subjektnahe Wirkungsforschung, Milieusstudien (wie sie im Felde der Bildung Michael Vester u. a., Leibniz Universität Hannover, vorlegen), Heranziehen von Künstlertheorien usw.

Da Theater/Performanz/Pädagogik Praxeologien sind, also hohe Tätigkeitsqualität haben, muss die Logik dieser ihrer Praxis gewürdigt werden: es werden Beschreibungsverfahren, Schreibpraxen benötigt - also Praxeographien - und aktuelle und reflexive theaterpädagogische Praxis-Publizistik ist vonnöten; sie besteht z. T. schon - siehe scenario, siehe SpielArt (http://www.spielartberlin.de/) - Ausbildungssegmente in den Fachdidaktiken wie in der Theaterund Kulturpublizistik fehlen weitgehend. Zu nennen wäre auch die Notwendigkeit der Herausbildung eines eigenen Rezensionswesens für gerade solche, Theater und Pädagogik und Didaktik/Methodik verbindenden Aktivitäten. (vgl. Jahnke 2003).

Einige Stichworte zum eben Benannten (zusammenfassend / weiterführend):

- Methoden des kreativen Schreibens können, wenn sie hierfür zugespitzt werden, hilfreich sein.

- Das Wort von einer poeto science findet sich neuerlich in der Fachliteratur.

- Meines Erachtens ist es nötig, Marketingstrategien für neue, in diesen Feldern passende Forschungsweisen zu entwickeln (die Form der Skandalisierung darf von Fall zu Fall angewendet werden, denke ich).

- Bestehender Kultur- und Wissenschaftsjournalismus wäre zu nutzen und $\mathrm{zu}$ verbessern für theatrale, szenische, theaterpädagogische Unternehmungen (einige Zeitschriften, Publikationen in Didaktik, Theaterpädagogik bestehen bereits).

- Wirkungsforschung geschieht aber auch durch Arbeit in und mit Fachverbänden, seien es schulfach-orientierte, pädagogische oder Theater orientierte. Es gibt seit Jahren eine „Ständige Konferenz Spiel und Theater an Hochschulen" (2011 tagte sie an der Sporthochschule in Köln; 2012 wird sie an der Hochschule Osnabrück, Standort Lingen, Institut für Theaterpädagogik, stattfinden).

- Aus einer Arbeitsgruppe während des Hamburger Kongresses der „Deutschen Gesellschaft für Erziehungswissenschaft" hat sich ein Netzwerk 
für Theater in der (Hochschul-)Lehre gebildet. Seine Ergebnisse wurden bisher in zwei Bänden veröffentlicht (vgl. Bülow-Schramm et al. 2008, Hentschel et al.2008).

- Nebenbei: für das Arbeitsfeld Sprache/Sprechen usw. wären zum Beispiel die Amateurtheater und ihre Verbände, die Mundart-Theater und Volkstheater, 'mit ins Boot zu nehmen' - etwa in Kooperation mit schulischem Unterricht...

- Probleme beim fachlichen Selbstverständnis: Ist etwa Theater ,nur Methode für andere gute Zwecke? Oder stecken im theatralen Vorgang selbst Bildungswerte?

- Eine spitze Zwischenbemerkung: Will manch ein Theaterpädagoge nicht eigentlich Regisseur sein/werden?! Auch das ein Problem....

- Verlage, Buchhändler, Bibliotheken haben ihrerseits Probleme: in welche Rubrik stellt man Publikationen aus solchen kombinierten Arbeitsfeldern ein? Kunst, Pädagogik, Theater, Fachdidaktik, Sozialpädagogik? Das Problem wiederholt sich bei der Drittmittelförderung: Wer, was sind die, die Theater(kunst) und z.B. Fachdidaktik verbinden? Wo müssen sie sich einordnen? Vorschlag: Mehr selbstbewusstes marketing für die neuen Sach/en, die (in welchen Berufsfeldern auch immer) gemacht werden! Keine Angst vor ,Instrumentalisierung' und ,Verzweckung'. Nur Mut also courage, was ja auch heißt: (zivile, fachliche) Tapferkeit zeigen und beherzt sein!

Was können Theaterleute? Sie können handeln, forschen und gestalten. Gegenüber den arbeitsteiligen, heutigen Wissenschaftszweigen hätten Theater/Pädagogik einen glücklichen Vorzug: Forschung hat Bildungswert! Theaterleute (im weiteren Sinne - also auch PädagogInnen, die sich und ihren Beruf ,theatralisieren') haben Methoden, um im Theater-Medium über sich und von sich zu handeln! Hier können Erkenntnisse gestaltet und, in Szene gesetzt werden. Und es können auch die Erkenntniswege, Interessen, Zwecke - und die Einsprüche anderer dagegen vorgezeigt werden - auf dem eigenen Schauplatz - dem teatron - und sie sind nicht weit weg von Wissenschaft; denn: teatron/teatrum und theoria/teoria haben denselben Wortstamm - nämlich schauen und wahrnehmen - und das durchaus von Interesse geleitet und nicht zweck-los ...

\section{Bibliografie}

Adorno, Theodor W. (1970): Ästhetische Theorie. Frankfurt am Main: Suhrkamp 
Appel, Michael; Schumann, Michael; Stötzel, Angelika (Projektgruppe WANJA) (Hrsg.) (2000): Handbuch zum Wirksamkeitsdialog in der Offenen Kinder- und Jugendarbeit. Münster: Votum

Arendt, Hannah (1993): Was ist Politik? München/Zürich: Piper

Asholt, Wolfgang; Ette, Ottmar (2010): Literaturwissenschaft als Lebenswissenschaft. Tübingen: Narr

Bieri, Peter (2011): Wie wollen wir leben? Salzburg: Residenz

Bloch, Ernst (1970): Atheismus im Christentum. Reinbek: Rowohlt

Brecht, Bertolt (1993): Schriften 2. Große kommentierte Berliner und Frankfurter Ausgabe, Bd. 22.2., Berlin/Frankfurt am Main/Weimar: Aufbau/Suhrkamp

Brecht, Bertolt (1992): Schriften 1. Große kommentierte Berliner und Frankfurter Ausgabe, Bd. 21., Berlin/Frankfurt am Main/Weimar: Aufbau/Suhrkamp

Brecht, Bertolt (1973): ARBEITSJOURNAL. Bd. 2: 1942-1955. Frankfurt am Main: Suhrkamp

Buck-Morss, Susan (2011): Hegel und Haiti. Für eine neue Universalgeschichte. Frankfurt am Main: Suhrkamp

Bülow-Schramm, Margret; Gipser, Dietlinde; Krohn, Doris (Hrsg.) (2008): Bühne frei für Forschungstheater. Oldenburg: Freire

Chawtassi, Grigorij (1972): Weltergänzung durch Poesie. In: Weimarer Beiträge, Heft 2, 145-161

Faber, Richard (2005): Der Collage-Essay. Eine wissenschaftliche Darstellungsform. Hommage à Walter Benjamin (2., nahezu unveränderte Aufl.). Frankfurt am Main: Seifert

Faber, Richard (1999): Merkprosa. Benjamins Erzähltheorie und der Erzähler Benjamin. In: Faber, Richard; Naumann, Barbara (Hrsg.): Literarische Philosophie - Philosophische Literatur. Würzburg: Königshausen \& Neumann, 253-274

Flasch, Kurt (2010): Dankesrede am 2. 5. 2010 bei der Verleihung des Lessing-Preises für Kritik in Wolfenbüttel - nach dem Rundfunk-Mitschnitt, Deutschlandfunk (transkribiert von GK)

Flick, Uwe; von Kardorff, Ernst; Steinke, Ines (Hrsg.) (2000): Qualitative Forschung. Ein Handbuch. Reinbek: Rowohlt

Flusser, Vilém (1991): Kleine Anthropologie der Dinge. Vom Hebel. In: Die Zeit 15. 3., $70-71$

Gad, Stefan (2010): Heterogenität als gesellschaftlicher Normalfall Partizipation statt Präsentation in Schule und lokalen Strukturen, unveröff. Vortrag, Berlin

Geertz, Clifford (1987): Dichte Beschreibung. Frankfurt am Main: Suhrkamp Glissant, Édouard (2005): Kultur und Identität. Ansätze zu einer Poetik der Vielheit. Heidelberg: Wunderhorn 
Goffman, Erving (1983): Wir alle spielen Theater. München: Piper

Han, Byung-Chul (2005): Hyperkulturalität. Berlin: Merve

Hentschel, Ingrid; Wildt, Beatrix; Wildt, Johannes (Hrsg. unter Mitarbeit von Gerd Koch) (2008): Theater in der Lehre. Verfahren, Konzepte, Vorschläge. Münster: Lit

Hilliger, Dorothea (2006): Theaterpädagogische Inszenierung. Berlin/Milow/Strasburg: Schibri

Jahnke, Manfred (2003): Rezensionen von theaterpädagogischen Aufführungen. In: Koch, Gerd; Streisand, Marianne (Hrsg.): Wörterbuch der Theaterpädagogik. Berlin/Milow/Strasburg: Schibri, 247-249

Kleve, Heiko; Koch, Gerd; Müller, Matthias (Hrsg.) (2003): Different und Soziale Arbeit. Berlin/Milow/Strasburg: Schibri

Knott, Marie Luise (2011): Verlernen. Denkwege bei Hannah Arendt. Berlin: Mathes \& Seitz

Koch, Gerd (2012): Das Erzähl-Café als Methode in der rekonstruktiven Sozialen Arbeit. In: Rätz, Regina; Völter, Bettina (Hrsg.) (erscheint): Wörterbuch Rekonstruktive Soziale Arbeit. Leverkusen: Budrich

Koch, Gerd (2008): Ungeschützte Bemerkungen zu „Creative Drama“ In: Spielart [http://www.spielart-berlin.de/2008/12/18/gerd-kochungeschutzte-bemerkungen/, 1.5.2012]

Koch, Gerd (2005): Lernen lernen - Lehren lernen - Lehren lehren. In: Welbers, Ulrich; Olaf Claus (Hrsg.): The Shift from Teaching to Learning. Bielefeld: Bertelsmann, 197-201

Koch, Gerd (2001): Die Kunst zu leben. Theatralisierung von Lehr- und Lernprozessen. Idee und Realisation: Laszlo Kornitzer (Video-Film $47 \mathrm{~min}$, Schnitt: Immo Schönborn). Berlin/Milow: Schibri

Koch, Gerd (1997): Theater-Spiel als szenische Sozialforschung. In: Jürgen Belgrad (Hrsg.): TheaterSpiel. Hohengehren: Schneider, 81-95

Koch, Gerd (ed.) (1995): Theatralisierung von Lehr-Lernprozessen. Milow: Schibri

Koch, Gerd (1986): Kulturkreis oder Multiversum? Blochs Beitrag zur Multikultur. In: KulturRevolution. Zeitschrift für angewandte Diskurstheorie 12, 14-15

Koch, Gerd; Manke, Winfried (1986): Mut zur Entschulung - eine Polemik. In: Claußen, Bernhard (Hrsg.): Texte zur politischen Bildung, Bd. 2. Frankfurt am Main: Haag + Herchen 235-244

Küppers, Almut; Schmidt, Torben; Walter, Maik (Hrsg.) (2011): Inszenierungen im Fremdsprachenunterricht. Braunschweig: Diesterweg/Klinkhardt

Lassak, Jens (2011): Das Theaterfeature. Analyse einer neuen Form der kritischen Auseinandersetzung mit politisch-historischen Bildungsinhalten gezeigt am Beispiel des Projekts „Stille Helden - Warum werden Menschen mutig?", unveröff. Bachelor-Arbeit an der Alice Salomon Hochschule Berlin 2011, Gutachter: Gerd Koch, Stephan Weßeling 
Lenakakis, Antonis (2003a): Paedagogus Ludens. Berlin/Milow: Schibri

Lenakakis, Antonis (2003b): Zur Wirkung der Spiel- und Theaterpädagogik auf Lehrer(innen). In: Korrespondenzen. Zeitschrift für Theaterpädagogik 43, 25-28

Lukács, Georg (1972): Ästhetik, Bd. 1. (2. Aufl.). Darmstadt/Neuwied: Luchterhand

Münz, Rudolf (1998): Theatralität und Theater. Berlin: Schwarzkopf \& Schwarzkopf

Narbutovic, Katharina; Stemmler, Susanne (Hrsg.) (2011): Über Lebenskunst. Utopien nach der Krise. Berlin: Suhrkamp

Negt, Oskar (1971): Soziologische Phantasie und exemplarisches Lernen (völlig überarb. Aufl.). Frankfurt am Main: EVA

Pfütze, Hermann (1999): Ursprung und Gegenwart der Kunst. Frankfurt am Main: Suhrkamp

Pigl, Michael; Feldtkeller, Franz; Koch, Gerd (2006): Beschreibende, mehrperspektivische Wirkungsforschung - gezeigt am Beispiel vom Kinderund Jugendzirkus Montelino. In: Zeitschrift für Theaterpädagogik 48, 65-69

Rancière, Jacques (1994): Die Namen der Geschichte. Frankfurt am Main. Suhrkamp

Rumpf, Horst (2010): Was hätte Einstein gedacht, wenn er nicht Geige gespielt hätte? Gegen die Verkürzungen des etablierten Lernbegriffs. Weinheim/ München: Juventa

Rumpf, Horst (1979): Worauf zu achten wäre - Aufmerksamkeitsrichtungen für die Friedenserziehung. In: Friedensanalysen. Für Theorie und Praxis 10. Frankfurt am Main: Suhrkamp, 161-165

Rumpf, Horst (1971): Scheinklarheiten. Sondierungen von Schule und Unterrichtsforschung. Braunschweig: Westermann

Projektteam Lokaljournalismus (ed.) (1986): ABC des Journalismus (neu strukturierte, aktualisiere und ergänzte 4. Aufl.). München: Ölschläger

Sack, Mira (2011): spielend denken: Theaterpädagogische Zugänge zur Dramaturgie des Probens. Bielefeld: transcript

Schanze, Helmut (ed.) (2001): Handbuch der Mediengeschichte. Stuttgart: Kröner

Sen, Armatya (2007): Die Identitätsfalle. Warum es keinen Kampf der Kulturen gibt. München: Hanser

Skirbekk, Gunnar (2002): Praxeologie der Moderne. Weilerswist: Velbrück

Taylor, Charles (1995): Das Unbehagen an der Moderne, Frankfurt am Main: Suhrkamp 